\title{
ERP evidence of preserved early memory function in term infants with neonatal encephalopathy following therapeutic hypothermia
}

\author{
Katie M. Pfister ${ }^{1,2}$, Lei Zhang ${ }^{3}$, Neely C. Miller ${ }^{1,2}$, Solveig Hultgren', Chris J. Boys ${ }^{1}$ and Michael K. Georgieff',2
}

BACKGROUND: Neonatal encephalopathy (NE) carries high risk for neurodevelopmental impairments. Therapeutic hypothermia (TH) reduces this risk, particularly for moderate encephalopathy (ME). Nevertheless, these infants often have subtle functional deficits, including abnormal memory function. Detection of deficits at the earliest possible time-point would allow for intervention during a period of maximal brain plasticity.

METHODS: Recognition memory function in 22 infants with NE treated with TH was compared to 23 healthy controls using event-related potentials (ERPs) at 2 wk of age. ERPs were recorded to mother's voice alternating with a stranger's voice to assess attentional responses (P2), novelty detection (slow wave), and discrimination between familiar and novel (difference wave). Development was tested at 12 mo using the Bayley Scales of Infant Development, Third Edition (BSID-III).

RESULTS: The NE group showed similar ERP components and BSID-III scores to controls. However, infants with NE showed discrimination at midline leads $(P=0.01)$, whereas controls showed discrimination in the left hemisphere $(P=0.05)$. Normal MRI $(P=0.05)$ and seizure-free electroencephalogram (EEG) $(P=0.04)$ correlated positively with outcomes.

CONCLUSION: Infants with NE have preserved recognition memory function after $\mathrm{TH}$. The spatially different recognition memory processing after early brain injury may represent compensatory changes in the brain circuitry and reflect a benefit of TH.

$\mathbf{N}$ eonatal encephalopathy (NE) affects $1-8 / 1,000$ term infants and carries a high risk for neurodevelopmental disabilities (1). While it is generally understood that those with mild encephalopathy do relatively well and those with severe encephalopathy have clear cognitive and motor deficits, a great deal of heterogeneity occurs in the outcome of infants with moderate encephalopathy (ME). Up to $75 \%$ of infants with ME have cognitive impairments without motor deficits or cerebral palsy (CP) $(2,3)$. This group has lower IQ at school age than their peers, delays in school readiness, and an increased risk of falling behind in school $(2,4,5)$. Their deficits are sometimes subtle, remaining undetected until school age when increasingly complex cognitive demands arise. Nevertheless, these deficits affect learning and daily functioning $(6,7)$.

Multiple studies done prior to therapeutic hypothermia (TH) showed that infants with ME had poorer working memory, long-term episodic memory, verbal and visual memory in middle childhood, and adolescence even when adjusting for IQ (2,7-9).

However, only one study has evaluated memory function in school age children after NE in the TH era. The authors found that children who had undergone $\mathrm{TH}$ had similar memory function to those who had not been cooled. As it was not the design of their study, they were unable to compare memory function to healthy controls or subdivide children by severity of encephalopathy (10).

While memory dysfunction at school age is easily tested through standardized neuropsychological tests, earlier detection would allow for intervention and therapy during the period of maximum brain plasticity that occurs during infancy. However, memory dysfunction is not easily detected in infants and toddlers through typical developmental assessments like the Bayley Scales of Infant Development (BSID), nor is it evident in daily functioning in this age group.

Studies of other at-risk populations have found memory dysfunction to be detectable in the newborn period using event-related potentials (ERPs) $(11,12)$. ERPs allow for early evaluation of specific brain processes in non-verbal infants by evaluating the brain's electrophysiologic response to specific stimuli using electroencephalogram (EEG). In newborn auditory recognition memory (a subcategory of declarative memory) ERPs, there are several well-described electrophysiologic components that index various aspects of memory function. The initial attentional response to the auditory stimulus is represented by an early wave peak $\sim 150-400 \mathrm{~ms}$ (P2). The complex waveform directly after P2 (hereafter referred to as "P2 complex") is thought to represent a more mature response to a two-syllable word $(13,14)$. The slow wave, which occurs after $\sim 1,000 \mathrm{~ms}$, represents cognitive processing of the stimulus 
(return near baseline $=$ familiarity, negative slow wave (NSW) = novelty detection); and the difference wave (the difference in mean amplitude of the slow wave between two different stimuli) represents central discrimination between stimuli most likely by the medial temporal lobe $(11,13)$. Thus, this technology has been used for early detection of typical and atypical memory function in at risk infants well before clinical performance is apparent $(11,12)$.

The aims of this study were to establish whether memory function could be assessed during the newborn period using ERP technology in infants with $\mathrm{NE}$ who had undergone $\mathrm{TH}$, to determine any differences in memory function between infants with NE and healthy controls, and to evaluate the relationship of newborn memory function with developmental status at 12 mo. We hypothesized that newborn memory function in this population would be worse than healthy control infants as measured by strength of the attentional response (specifically, a decreased P2 amplitude), as well as the ability to discriminate between stimuli (smaller difference wave), and that there would be a significant positive relationship between newborn memory function and development at $12 \mathrm{mo}$.

\section{RESULTS}

Forty-five infants with artifact-free data were included in the newborn data analysis: 22 infants in the NE group and 23 infants in the control group. Descriptive characteristics are shown in Table 1. Many of the noted perinatal factors have been shown to affect neurodevelopment (15-17), and more specifically infant ERP in the case of maternal diabetes (11). Of note, most infants had $\mathrm{ME}(n=17)$-only 1 had severe encephalopathy, 3 had mild encephalopathy, and one infant did not have a Sarnat score documented. There were missing data in each of the following categories (each missing from only one infant, no infant was missing data in multiple categories): delivery mode, infant of diabetic mother (IDM) status, Sarnat score, Seizure/EEG data, 1 min Apgar, $5 \mathrm{~min}$ Apgar. Infants in the two groups differed in 1 - and 5 -min Apgar scores $(P \leq 0.01)$, maternal age $(P \leq 0.01)$, maternal education $(P=0.05)$, maternal race $(P \leq 0.01)$, and $\operatorname{diet}(P=0.015)$. Twelve-month follow-up was completed by $18 / 22$ infants with NE and $22 / 23$ control infants. There was no significant difference in characteristics between the infants lost to follow-up and those who underwent 12-mo evaluation. For the newborn ERPs, there was no significant difference in the average number of acceptable (artifact-free) trials between stimulus type or study groups.

Grandmean ERPs for auditory recognition memory are shown in Figure 1. Infants in both groups showed similar waveforms with a P2 response to both mother's (familiar) and stranger's (novel) voices, implying intact attentional neural circuitry. Like controls, the NE group also had a complex waveform after P2 with new peaks arising in the midportion of the ERP (P2 complex). Each group had NSWs to the novel stimulus and significant difference waves, implying intact discrimination between stimuli; however, the location of discrimination differed between groups.
Table 1. Infant characteristics

\begin{tabular}{|c|c|c|c|}
\hline Newborn characteristic & $\begin{array}{c}\text { Neonatal } \\
\text { encephalopathy } \\
(N=22)\end{array}$ & $\begin{array}{l}\text { Control } \\
(N=23)\end{array}$ & $P$ value \\
\hline Sex-male n (\%) & $14(63.6)$ & $12(52.2)$ & 0.55 \\
\hline Birth gestation age-weeks (SD) & $39.9(1.1)$ & $39.5(1.0)$ & 0.19 \\
\hline Birth weight—kg (SD) & $3.74(0.52)$ & $3.57(0.38)$ & 0.23 \\
\hline Age at first visit—weeks (SD) & $2.98(0.84)$ & $2.76(0.69)$ & 0.33 \\
\hline $\begin{array}{l}\text { Corrected gestational age at } \\
\text { visit—weeks (SD) }\end{array}$ & $42.9(1.4)$ & $42.3(1.4)$ & 0.13 \\
\hline Mode of delivery-Cesarian n (\%) & $7(31.82)$ & $11(50.00)$ & 0.36 \\
\hline Diet—n (\%) Breast milk—n (\%) & $11(50.00)$ & $20(86.96)$ & 0.02 \\
\hline Formula $-n(\%)$ & $2(9.09)$ & $1(4.35)$ & \\
\hline Both-n (\%) & $9(40.91)$ & $2(8.70)$ & \\
\hline IDM-n (\%) & $2(9.09)$ & $4(18.18)$ & 0.66 \\
\hline Mother's age-years (SD) & $29.59(4.99)$ & $34.30(4.98)$ & $<0.01$ \\
\hline Mother's race-white race $n(\%)$ & $15(68)$ & $23(100)$ & $<0.01$ \\
\hline $\begin{array}{l}\text { Mother's highest education-more } \\
\text { than high school } n(\%)\end{array}$ & $18(82)$ & $23(100)$ & 0.05 \\
\hline 1 min Apgar (SD) & $1.57(1.03)$ & $7.87(1.71)$ & $<0.01$ \\
\hline 5 min Apgar (SD) & $3.71(2.05)$ & $8.91(0.29)$ & $<0.01$ \\
\hline Sarnat score (SD) & $1.9(0.4)$ & & \\
\hline Initial pH (SD) & $7.0(0.21)$ & & \\
\hline Initial base excess (SD) & $-17.6(4.7)$ & & \\
\hline MRI abnormal- $n(\%)$ & $11(50)$ & & \\
\hline EEG abnormal-n (\%) & $8(38)$ & & \\
\hline Seizures-n (\%) & $7(33)$ & & \\
\hline
\end{tabular}

\section{Attentional Response (P2)}

There was no significant difference in peak P2 amplitudes between $\mathrm{NE}$ and control groups in any scalp location or when all scalp locations were combined. Infants with $\mathrm{NE}$ had a higher P2 to familiar compared to novel stimuli at left ( $P=$ 0.03 , Cohen's $\mathrm{d}=0.5)$, midline $(P=0.02, \mathrm{~d}=0.55)$, and overall $(P=0.05, \mathrm{~d}=0.4)$. Control infants showed no significant difference in P2 amplitude between stimuli. (Figure 2)

Infants with NE had longer latency to $\mathrm{P} 2$ to familiar voice vs. novel at left $(P=0.01, \mathrm{~d}=0.6)$ and overall $(P=0.02, \mathrm{~d}=0.4)$. Control infants did not show any differences in $\mathrm{P} 2$ latencies in any area to familiar vs. novel. There was also no significant difference in latency between NE vs. control groups in any area.

Because a large portion of our infants with NE had moderate encephalopathy, further analysis was done on only those infants. Infants with ME had a higher P2 to familiar compared to novel stimuli at left with borderline significance $(P=0.07$, $\mathrm{d}=0.5)$ and significantly at midline $(P=0.05, \mathrm{~d}=0.5)$. They also had longer latency to familiar versus novel at left $(P=0.04$, $\mathrm{d}=0.6)$.

\section{P2 Complex}

Control infants had higher mean amplitude of the P2 complex to familiar stimuli at midline $(P=0.03, \mathrm{~d}=0.4)$ and trended toward significance in the left hemisphere $(P=0.06, \mathrm{~d}=0.4)$. 
a
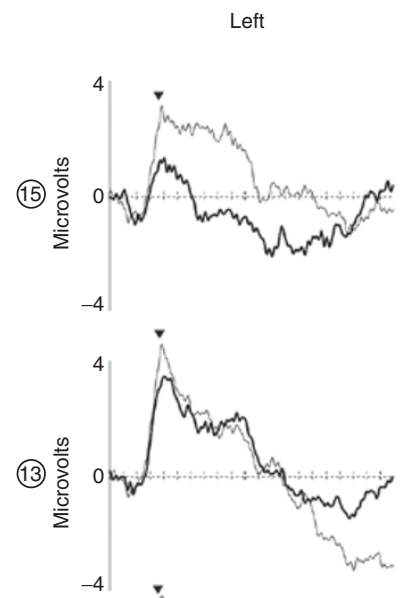

(9)

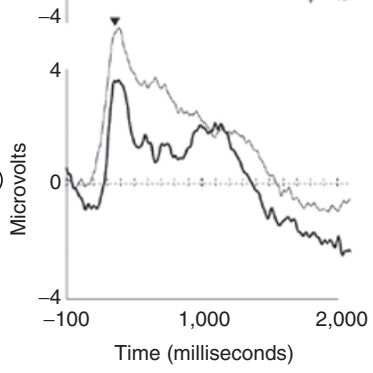

b

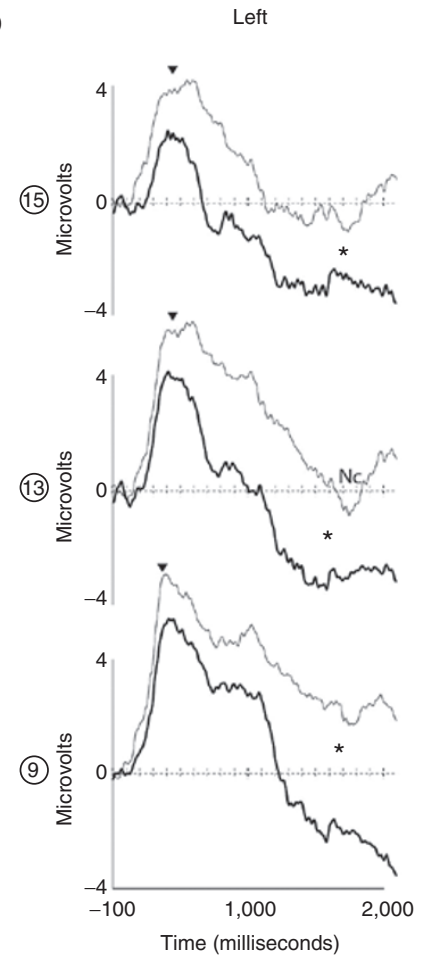

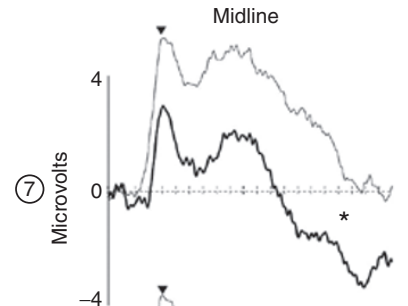

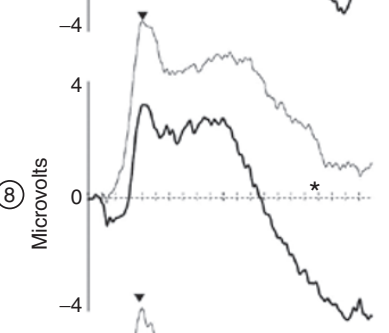

(3)
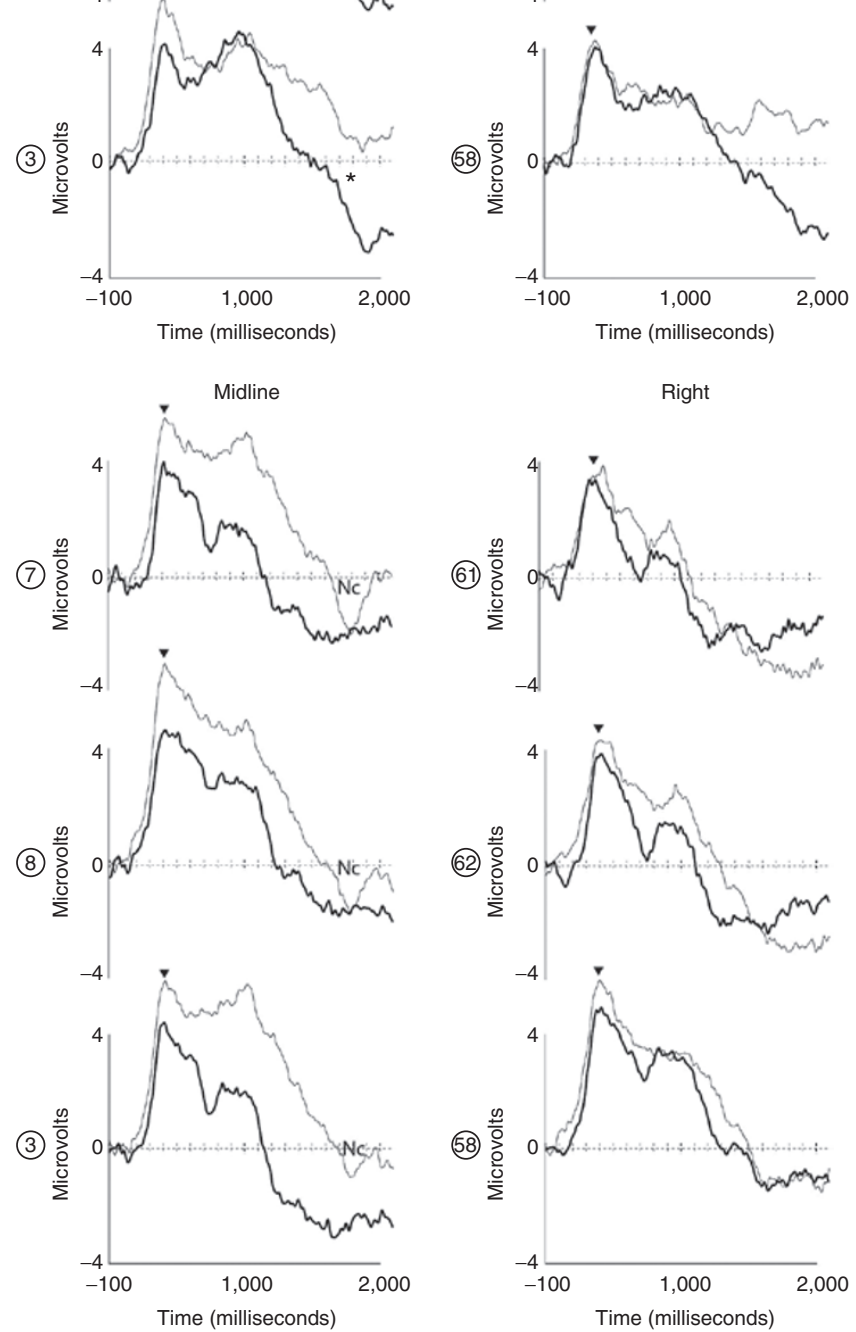

Right
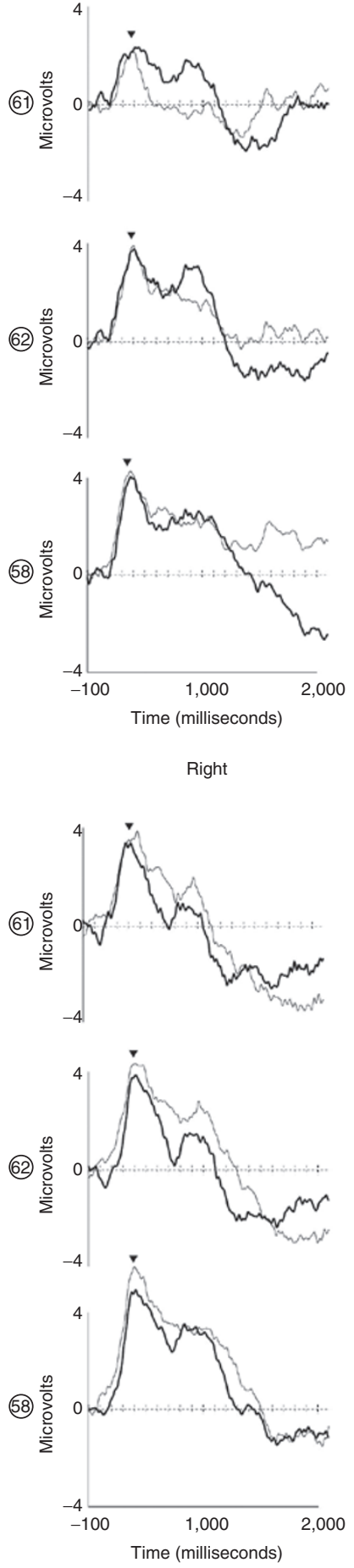

Figure 1. Grandmean event-related potentials (ERPs). Each column contains the grandmean ERPs from the three electrodes analyzed in that scalp region cluster (electrode number in circle at left of graphic). (a) Left, midline and right grandmean ERPs from infants with NE in response to familiar (thin line) and novel (thick line) auditory stimuli. (b) Left, midline and right grandmean ERPs from control infants in response to familiar (thin line) and novel (thick line) auditory stimuli. Arrow heads denote P2. ${ }^{*}$ significant difference wave when that cluster of leads is averaged. Nc, negative central. Note P2 complex from $600-1,000 \mathrm{~ms}$ in both groups.

Infants with NE did have a P2 complex, however, they had no difference in P2 complex amplitude between stimuli and there was no difference between the amplitudes of NE vs. control groups.

\section{Novelty Detection (NSW)}

Infants with NE showed novelty detection with a NSW to novel stimuli and showed discrimination between the two types of stimuli at midline $(P=0.01, \mathrm{~d}=0.6)$, as shown by 
the difference in amplitude of the slow wave to each stimulus. Subanalysis of only infants with ME also showed discrimination between stimuli at left $(P=0.03, \mathrm{~d}=0.6)$. Control infants showed novelty detection and discrimination between the two types of stimuli in the left hemisphere $(P=0.05, \mathrm{~d}=0.4)$. Although controls had a NSW to the novel stimulus at midline leads, the mean amplitude was not significantly different from the slow wave for the familiar stimulus. There was no difference between study groups in the size of the difference wave in any region (Figure 3 ).

\section{Effect of Clinical Factors on Attentional Response and Stimulus Discrimination in Infants With NE}

Infants with moderate/severe encephalopathy showed a longer P2 latency to familiar compared to stranger $(P=0.04, \mathrm{~d}=0.5)$; there was no difference in latency between stimuli in those with mild encephalopathy compared to those with moderate/ severe.

Focusing on midline leads, where infants showed discrimination between stimuli (see above), infants without seizures and infants with normal MRIs showed discrimination between stimuli based on the difference wave $(P=0.04, \mathrm{~d}=0.6$ and $P=0.05, \mathrm{~d}=0.6$, respectively), whereas infants with seizures or abnormal MRIs did not show significant discrimination. Infants with abnormal EEGs (either abnormal baseline that later normalized, or seizure activity) did show discrimination between stimuli $(P=0.04, \mathrm{~d}=0.9)$. Infants with moderate or severe encephalopathy showed discrimination between stimuli $(P=0.03, \mathrm{~d}=0.6)$. Those with mild NE did not show discrimination $(P=0.25)$; however the analysis was likely underpowered since were only three infants in this group (Table 2). Base excess and $\mathrm{pH}$ were not significantly associated with the difference wave.

\section{Twelve-Month Follow-Up}

In an unadjusted analysis, control infants had higher language scores on the BSID-III than infants with NE $(P=0.04, \mathrm{~d}=0.7)$. However, after adjusting for mother's age, race, education, and infant's diet (all known to be associated with development (1517)), there were no significant differences in BSID-III scores in any of the domains between infants with NE and controls (Table 3). In a subanalysis comparing infants with ME to controls, there were no significant differences in BSID-III scores.

Using Pearson correlation coefficients and the covariates mentioned above, there was no association between the size of the difference wave at the newborn visit and the 12-mo BSIDIII score in either group. The P2 latency was negatively correlated with the language score in the control group $(P=0.02)$ but not in the NE group.

\section{DISCUSSION}

Early detection of memory function in healthy and at risk term infants is feasible using ERP, and this group of infants with NE showed preserved recognition memory function following $\mathrm{TH}$. Subanalysis of just those infants with ME, the group known to have the most heterogeneous outcomes from NE, also shows

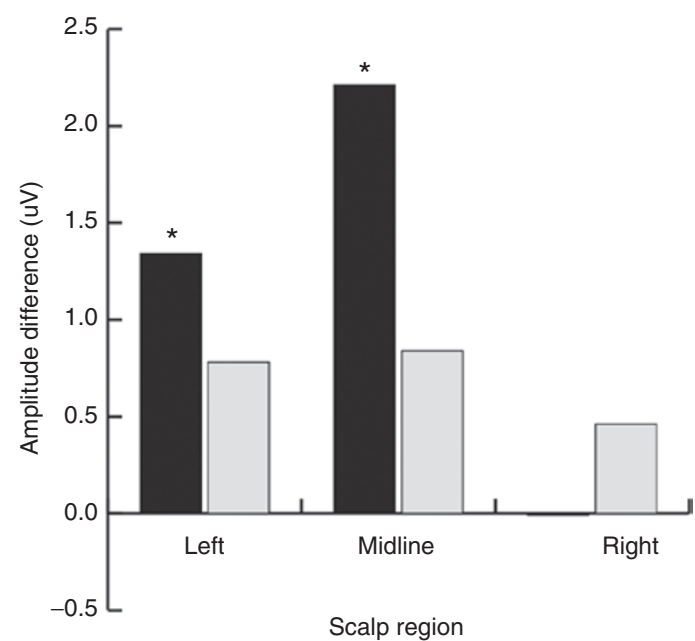

Figure 2. Difference in P2 amplitude (familiar-novel) by scalp region. Dark gray bars represent the neonatal encephalopathy group; light gray bars represent the control group. ${ }^{*} P<0.05$.

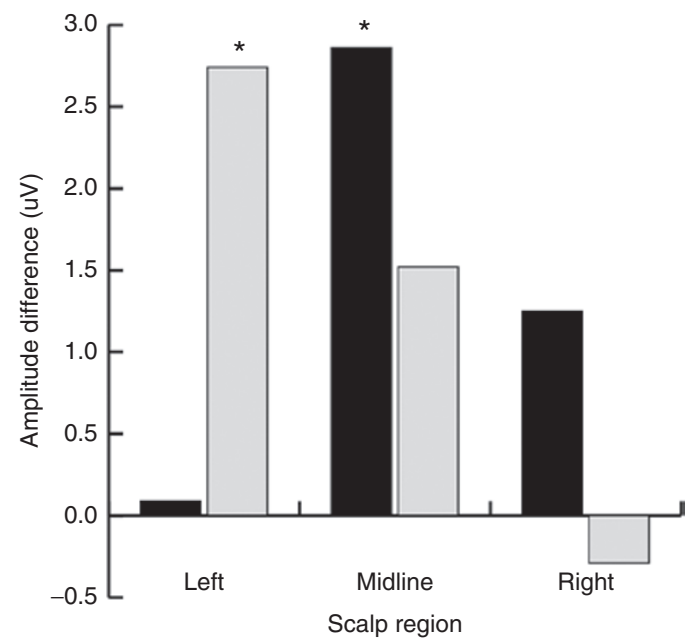

Figure 3. Size of difference waves (familiar-novel) by scalp region. Dark gray bars represent the neonatal encephalopathy group; light gray bars represent the control group. ${ }^{*} P \leq 0.05$.

Table 2. Association between difference wave (DW) at midline and clinical factors

\begin{tabular}{lcrcc}
\hline Variable & Category & $N$ & Mean DW (SD) & $P$ value \\
\hline EEG normal & Yes & 13 & $0.90(2.81)$ & 0.27 \\
\multirow{3}{*}{ Seizures } & No & 8 & $6.35(6.38)$ & 0.04 \\
& Yes & 7 & $4.92(7.58)$ & 0.30 \\
MRI normal & No & 14 & $2.00(3.30)$ & 0.04 \\
& Yes & 11 & $3.02(5.22)$ & 0.05 \\
SARNAT & No & 11 & $2.71(5.09)$ & 0.15 \\
& Mild & 3 & $1.46(1.34)$ & 0.25 \\
& Moderate/severe & 18 & $3.23(5.50)$ & 0.03 \\
\hline
\end{tabular}

preserved memory function. Further, the infants with NE had BSID-III scores that were not different from controls, suggesting a successful outcome of TH. The newborn ERP could be an early marker of later intact function. Although there did not 


\section{Articles $\mid$ Pfistere al.}

Table 3. Comparison of adjusted BSID-III scores ${ }^{\mathrm{a}}$ between groups

\begin{tabular}{lccc}
\hline Variable & $\begin{array}{c}\text { Neonatal } \\
\text { encephalopathy }\end{array}$ & Control & $P$ value \\
\hline Cognitive & $105.6(5.09)$ & $112.9(6.06)$ & 0.15 \\
Language & $100.5(5.86)$ & $101.7(6.98)$ & 0.84 \\
Motor & $100.9(6.43)$ & $109.0(7.66)$ & 0.20
\end{tabular}

aScores (mean (SE)) are adjusted for the following covariates: mother's age, mother's race, mother's education, infant diet.

seem to be any difference in "robustness" of memory function between infants with $\mathrm{NE}$ and controls, there were differences in the location of function in infants with NE from an electrophysiological standpoint suggesting that the circuitry has been reorganized to preserve the function.

Infants with NE showed a differentiation in attentional response to familiar stimuli compared to novel. While our control infants did not show any statistical differences in P2 between stimulus type, the $\mathrm{P} 2$ patterns of our infants with NE were similar to healthy newborns with more than $1 \mathrm{wk}$ of ex-utero "experience" in other studies $(11,13)$. This included higher amplitude and longer latency to P2 to familiar stimuli, as well as the P2 complex. The complex waveform after P2 is thought to represent more depth of processing of the word "baby", with the response to the second syllable of the word superimposed over the initial attentional response (13). Along with the attentional response, the infants with NE had a similar strength of discrimination between familiar vs. novel stimuli compared to controls after $\mathrm{TH}$, as shown by the difference wave, suggesting preservation of memory function.

Neonatal hypoxia-ischemia, one cause of NE, has been shown to lead to hippocampal damage and eventual memory dysfunction $(18,19)$. This was recently demonstrated in a study of 40 children with presumed neonatal hypoxia (that did not undergo TH) who subsequently had mean hippocampal volumes and memory scores significantly below normal controls. Further, the memory quotients were significantly below those predicted by the full-scale IQs (19). Despite these findings, the infants in our study had preserved recognition memory function, which could be a result of TH. Thus far, TH has been shown to improve cognitive outcomes in infants with NE, particularly those with ME $(10,20)$. A recent mouse-model study of mild-to-moderate hypoxia-ischemic (HI) injury showed that neural stem and progenitor cells (NSPCs), which are critical for postnatal development, normal adult hippocampal function, and facilitation of recovery after injury, are acutely decreased after $\mathrm{HI}$ in the dentate gyrus. This was followed by a decrease in neuroblasts and immature neurons $1 \mathrm{wk}$ postevent. However, mice treated with TH showed partial protection of the NSPCs and cell density (21).

Although the TOBY trial (10) did not show a difference in memory at school age between infants who were treated with $\mathrm{TH}$ and those who were not, that study did not separate out moderate versus severely affected infants for this portion of the testing. It is possible that the benefit of $\mathrm{TH}$ on memory function is predominantly seen in those with ME, the predominant group in our study. Thus, the beneficial effect is not seen when severity groups are pooled. In a meta-analysis of outcomes at 18 mo after hypothermia for NE, the effect of cooling seems to benefit those with ME more than those with severe encephalopathy with a relative risk reduction of $33 \mathrm{vs.} 17 \%$ in moderate vs. severe encephalopathy, respectively (20).

Infants with NE in our study showed similar strength of discrimination between familiar versus novel stimuli compared to controls. However, the two groups displayed different patterns of activation: the infants with NE showed auditory discrimination at midline leads whereas the control infants showed discrimination in left hemisphere leads. Lateralization of cognitive processes, including language, is a key characteristic of the developing brain (22), and neuroimaging and optical topography studies have shown left hemispheric dominance for auditory language even in newborns $(23,24)$. Kwon et al. showed that preterm infants have altered functional organization of the brain with less lateralization in regions subserving both expressive and receptive language at term corrected age compared to term controls using voxel-based connectivity analysis (25). Based on emerging research linking functional connectivity, blood supply, and metabolic demand $(26,27)$, the authors suggested that the decreased lateralization may index deficits in blood flow or metabolism in injured areas of the brain (25). This theory would explain the lack of lateralization in our infants who may have suffered an $\mathrm{HI}$ event, particularly those with moderate $\mathrm{NE}$ without $\mathrm{CP}$, which is thought to be caused by watershed injury $(3,28)$. Although the change in lateralization may reflect damage, it may also reflect a compensatory change allowing preserved memory function.

One other feature seen in the ERPs of our control infants was a negative deflection $\sim 1,800 \mathrm{~ms}$ (i.e., negative central $(\mathrm{Nc})$ ) at the very end of the ERP segment to their mother's voice, predominantly in the midline leads (Figure 1b). These are typically seen in older infants in visual recognition memory paradigms, especially oddball paradigms (29). In paradigms where two stimuli are presented with equal probability (like ours), the $\mathrm{Nc}$ is larger for the familiar stimulus (29). We did not see an Nc to the novel stimuli, possibly because the timing overlapped with the NSW to that stimulus. The Nc may also have affected the significance of the difference wave for the controls at midline leads because the mean slow wave amplitude is affected by that deflection; these infants may indeed be discriminating between stimuli at both midline and left hemisphere leads.

Early markers that predict long-term neurodevelopment are beneficial not only to identify those infants who might benefit most from early therapy/intervention (when the brain is still developing), but also to answer parental and caregiver questions about prognosis in at-risk infants. Several clinical features in the newborn period seemed to be predictive of newborn memory function in this study. Infants without seizures and infants with normal MRIs showed discrimination between stimuli. Others have also found seizures and MRI to be modest predictors of neurodevelopmental outcome (30-32). Surprisingly, infants with abnormal EEGs (either abnormal baseline that later normalized, or seizure activity) also showed 
discrimination between stimuli. While this seems counterintuitive, this again may be affected by the small sample size, as we were not able to separate out those infants who had quickly normalizing EEGs from those who had prolonged abnormality or seizures. Further, studies of EEG and brain imaging in infants with NE have had inconsistent results with regard to outcome predictability. Amplitude integrated EEG seems to be less predictive in infants who have received $\mathrm{TH}$ than those that did not (33-35). While seizure activity and MRI showed some prediction of early memory function, longer follow-up is needed to evaluate long-term predictive value. Evaluation of early memory function by ERP itself may be a more specific predictor of long-term outcome than general neurophysiologic and imaging modalities, as well as behavioral testing, as has been shown in other at-risk populations $(36,37)$.

There was no significant difference in BSID-III scores between groups after adjusting for confounding factors, nor was there correlation between size of the difference wave and neurodevelopmental scores. While it is encouraging that this group of infants with NE scored within the normal range on the BSID-III, and the early ERP data also suggests normal function, a 12-mo BSID-III is not necessarily predictive of long-term outcomes and future studies could evaluate whether newborn ERP data correlates with longer-term neurodevelopmental outcomes.

A major strength of this study was the ability to test memory function at such an early age. This allows for insight into early brain development without the effects of confounding factors that come with age including nutrition, home environment, and education. Other studies of memory function require an actively participating child or a child who is able to sit through an fMRI. ERPs are noninvasive and do not require sedation or behavioral responses from infants, making them an ideal mode for studying newborns. One potential study limitation is the small sample size, which could affect the power of the study. However, the sensitivity of ERPs allows for relatively small sample sizes to be used in neonates to detect important differences between groups (11-13). Another potential study limitation is the homogeneity of the NE group (mostly ME), which was driven by the inclusion criteria for cooling and those who could undergo testing in an outpatient setting early in the newborn period. However, this is the group of infants with NE with the most variability in outcome in whom early markers of outcome might be most beneficial. While the homogeneity did allow us to do a sub-analysis and make conclusions on a group of infants with ME, it would be beneficial to study early memory function in survivors with mild and severe NE, as well. This is particularly true for mild NE, as there is growing evidence that these infants do not have normal development, yet fall somewhere between controls and those with ME (38).

Other limitations are differences in characteristics between the two groups of infants that have been shown to potentially impact neurodevelopment and neural functioning. One factor is the altered ex-utero environment (NICU) that the infants with NE experienced. Much of the research in this area focuses on preterm infants with extended NICU hospitalizations (39). Our infants with NE were born at term gestation. As term infants, the systems we were assessing by ERP had matured in the appropriate (intrauterine) environment, where as preterm infants are maturing in an abnormal (extrauterine) environment. Moreover, our population had relatively brief NICU stays of $<2 \mathrm{wk}$ (and therefore relatively briefer exposures to the NICU environment compared to preterm infants). Nevertheless, it is possible that there was some environmental effect on their brain development. Although, it would be extremely difficult to find a control population of infants with an equal amount of time spent in the NICU without other confounding factors that affect neurodevelopment. The NICU stay could also have affected the infants' experience with the maternal voice; familiarity with the mother's voice was not controlled for. While the NE group may have had less postnatal experience with their mother's voice at the time of testing compared to controls due to hospitalization, previous studies have shown that term infants show recognition of their mother's voice via ERP soon after birth $(<2 \mathrm{~d})$ (13). This suggests minimal ex-utero exposure to the mother's voice is needed for recognition, and perhaps in-utero exposure alone is sufficient. Further, our infants in both groups showed a complex P2 wave, which has been shown to be present in term infants with "post-natal experience" of $>1$ wk (13), suggesting that the group with NE had sufficient experience with their mother's voice ex-utero, and likely comparable to the control infants.

Second, there was a difference in the percentage of infants receiving breast milk between groups. An exclusive breast milk diet has been associated with faster speed of processing of both auditory and visual stimuli compared with a formula diet in infants (40). Older infants (6 mo) also show hemispheric differences in ERP to speech stimuli based on diet (41). In these studies, the groups received exclusive breast milk or formula. In our study, the control group had significantly more infants receiving exclusive breast milk, which could potentially affect ERP data; however, the NE group had a larger number of infants receiving some breast milk. Thus, the number of infants receiving any breast milk did not differ between groups, so it would be difficult to conclude that diet had any affect on our results.

Future studies should focus on long-term effects of NE and $\mathrm{TH}$ on memory function, including the predictive value of early memory function. Kwon et al's study showed lack of late postnatal influence on lateralization-neither gestational age at birth, nor postmenstrual age at the time of the study influenced lateralization (25). Electrophysiologic analysis of our group of infants at an older age would shed light on the evolution of lateralization after an acute event.

\section{Conclusion}

Our study shows that recognition memory function is preserved in infants with $\mathrm{NE}$ who received $\mathrm{TH}$, though there is alteration of the normal lateralization process of brain development. This may be compensatory and a result of the benefit of therapeutic hypothermia. 


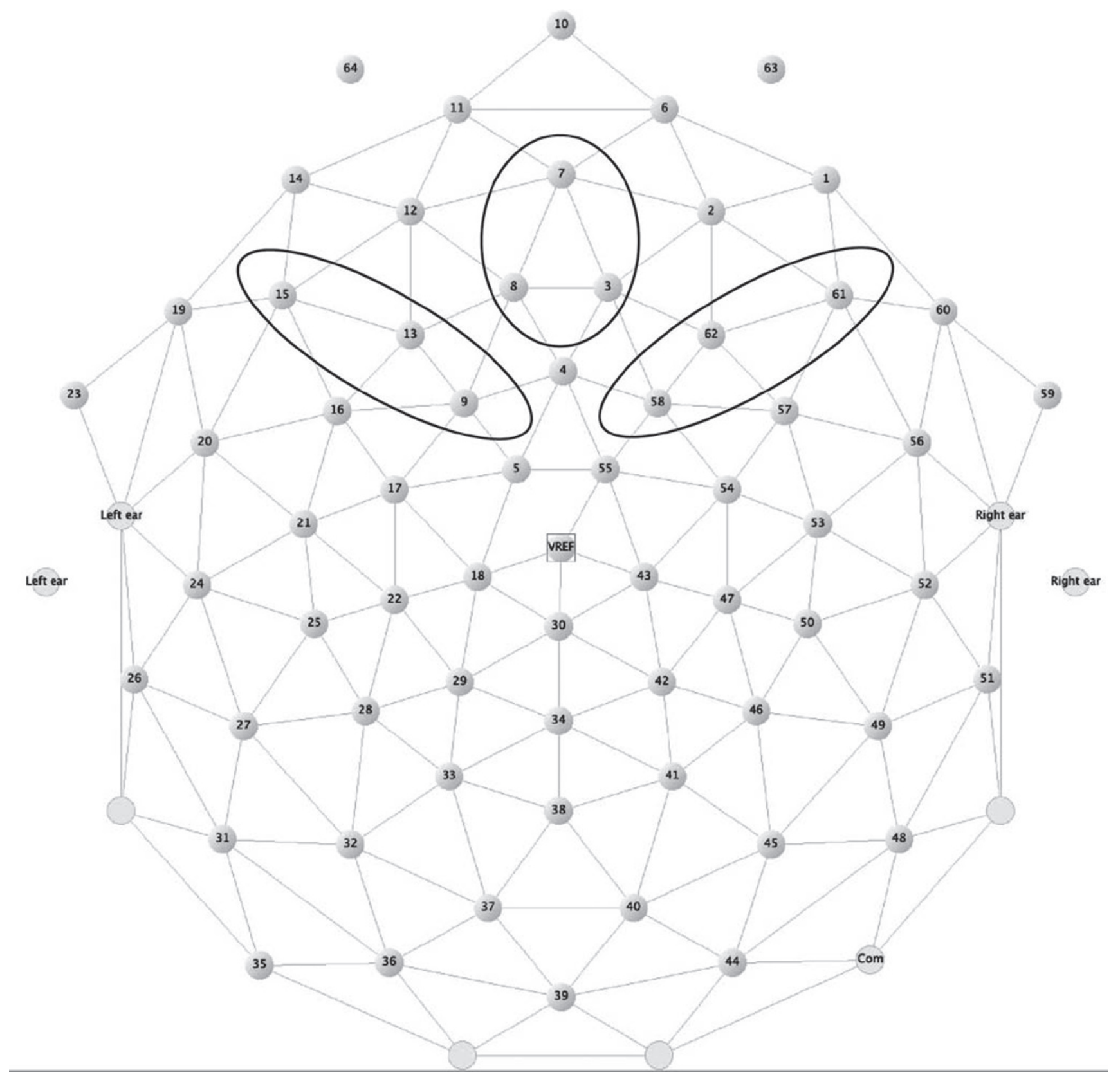

Figure 4. A schematic of the 64-channel geodesic sensor net (Netstation, EGI) and the clusters of electrodes used for data analysis.

\section{METHODS}

\section{Subjects}

The study group participants were recruited from the University of Minnesota Masonic Children's Hospital, Children's Hospitals and Clinics of Minnesota-St. Paul, and North Memorial Medical Center's neonatal intensive care units and the control group from the newborn nursery at the University of Minnesota Medical Center from 2011-2014. The Institutional Review Boards at the University of Minnesota, Children's Hospitals and Clinics of Minnesota, and North Memorial Medical Center approved the study and parents provided written consent for the participation of their infants.

All participants were $\geq 36 \mathrm{wk}$ gestational age at birth. Study group infants were eligible if they met criteria for and underwent therapeutic hypothermia due to neonatal NE (criteria similar to currently published guidelines (42) and based on Sarnat staging (43)) and were discharged by $4 \mathrm{wk}$ of age. Control group infants were required to have a 5 -min Apgar $\geq 7$ and remain healthy after birth. Exclusion criteria for both groups were the presence of a congenital abnormality or metabolic disease that would affect neurodevelopment, birth weight small for gestational age, or failure (or referral) on the routine newborn hearing screen done per institution guidelines. Twenty-three infants with NE and 25 control infants met criteria and had parental consent for participation.

At the first study visit, between $2-4 \mathrm{wk}$ of age, infants underwent assessment of auditory recognition memory via event ERP (described below) at the Center for Neurobehavioral Development (CNBD) at the University of Minnesota. At 12 mo infants returned for general neurodevelopmental assessment via the Bayley Scales of Infant Development, Third-Edition (BSID-III). Birth history and factors related to severity of illness during hospitalization were collected by chart review. Infant diet and parental age, race and highest education achieved were collected by parental self-report at the first visit.

\section{Newborn Auditory Recognition Memory Assessment}

Infant auditory recognition memory was assessed by recording ERPs in response to the maternal voice (familiar) randomly alternating 
with a stranger's voice (novel). The stranger's voice was the mother from the previously tested infant. Each stimulus type (familiar/novel) was presented 50 times per testing session. Interstimulus interval was $2500 \mathrm{~ms}$. The auditory stimulus was the word "baby" digitized and edited to $750 \mathrm{~ms}$ and $70 \mathrm{~dB}$ with Creative WaveStudio 5.00 .10 software (Creative Technology, Singapore, Republic of Singapore). The stimuli were presented via E-prime software (Psychology Software Tools, Sharpsburg, PA) through two speakers located 36 inches from the cranial end of the infant. The infant was swaddled and placed supine on a pillow on a parent's lap.

ERPs were recorded from ongoing EEG using the Geodesic EEG System 200 (Electrical Geodesics Incorporated (EGI), Eugene, OR) and a sixty-four channel sensor net designed in a "geodesic" pattern. Nets were available in a number of sizes appropriate for varying newborn head circumferences; a specific electrode is placed at the scalp vertex allowing for consistent placement of the electrodes on the scalp of each infant. Scalp impedances were measured using NetStation 4.4.2 software (EGI) and were accepted if $<50 \mathrm{KU}$. EEG was referenced to the vertex, amplified with a $0.1-100-\mathrm{Hz}$ bandpass and digitized at $250 \mathrm{~Hz}$.

\section{Data Analysis}

Data were later analyzed using NetStation 4.4.2 analysis software (EGI) using CNBD ERP analysis protocol (44) (described below). Data were filtered with a $30-\mathrm{Hz}$ lowpass filter, segmented to $2,100 \mathrm{~ms}$ periods starting $100 \mathrm{~ms}$ before stimulus presentation, and baseline corrected to the average prestimulus voltage. EEGs were hand-edited for poor recordings, movement artifact, and eye movement. Trials were excluded if the number of electrodes rejected was $>16 \%$. For acceptable trials, electrodes with bad data were replaced using spherical spline interpolation. Participants with $<12$ good trials per stimulus were excluded from further analysis. One infant with NE and two control infants were excluded based on inadequate trial number. For the remaining 23 controls and 22 infants with NE, the average waveform at each electrode was calculated and rereferenced to the average reference. We analyzed the most commonly reported components of auditory recognition memory ERPs $(11-14,45)$. P2 peak amplitude and latency were measured from a $150-450 \mathrm{~ms}$ window poststimulus. The mean amplitude of the $\mathrm{P} 2$ complex was measured from $600-1,000 \mathrm{~ms}$ poststimulus. The mean amplitude of the slow wave was measured from $1,200-2,000 \mathrm{~ms}$ poststimulus. We analyzed mean data from a fronto-central cluster of electrodes that showed the most activity to the stimuli. This cluster was consistent with the area of activity in other ERP studies of newborn auditory memory (11-14). The cluster was then divided into three regions (left, midline, right), each comprised of three electrodes (Figure 4). Per the manufacturer (EGI), the net's electrodes in these regions correspond approximately with the following locations on the 10-10 system: left (electrodes 15, 13, 9) corresponds approximately with F3, F5, FC1, midline (electrodes 7, 8, 3 ) corresponds approximately with $\mathrm{AFz}$ and $\mathrm{Fz}$, and right (electrodes $61,62,58)$ corresponds approximately with F6, F4, FC2.

Infant characteristics were summarized by groups. For between group comparisons, $P$ value was calculated by two-sample $t$-test for continuous variables and Fisher's exact test for categorical variable. For within group comparison (i.e., difference between familiar and novel voice), paired $t$-test was used. We did not control for multiple comparisons. For subgroup analysis by illness factors, Wilcoxon signed rank test was used to compare ERP data between familiar and novel voices. Linear regression was used to compare BSID-III scores between the NE and control groups adjusting for mother's age, race, education and infant diet. Pearson Correlation Coefficient was reported for association between difference wave and BSID-III score. A $P$ value of $<0.05$ was considered statistically significant. All analyses were performed in SAS (version 9.3; SAS Institute, Cary, NC) and graphs were plotted in $\mathrm{R}$.

\section{STATEMENT OF FINANCIAL SUPPORT}

This study was supported by the Center for Neurobehavioral Development, University of Minnesota.

Disclosure: The authors have no conflicts of interest to disclose related to this study.

\section{REFERENCES}

1. Kurinczuk JJ, White-Koning M, Badawi N. Epidemiology of neonatal encephalopathy and hypoxic-ischaemic encephalopathy. Early Hum Dev 2010;86:329-38.

2. Lindström K, Lagerroos P, Gillberg C, Fernell E. Teenage outcome after being born at term with moderate neonatal encephalopathy. Pediatr Neurol 2006;35:268-74.

3. Gonzalez FF, Miller SP. Does perinatal asphyxia impair cognitive function without cerebral palsy? Arch Dis Child Fetal Neonatal Ed 2006;91: F454-9.

4. Robertson CM, Finer NN, Grace MG. School performance of survivors of neonatal encephalopathy associated with birth asphyxia at term. J Pediatr 1989;114:753-60.

5. Robertson CM, Finer NN. Educational readiness of survivors of neonatal encephalopathy associated with birth asphyxia at term. J Dev Behav Pediatr 1988;9:298-306.

6. Gadian DG, Aicardi J, Watkins KE, Porter DA, Mishkin M, VarghaKhadem F. Developmental amnesia associated with early hypoxicischaemic injury. Brain 2000;123 Pt 3:499-507.

7. Mañeru C, Junqué C, Botet F, Tallada M, Guardia J. Neuropsychological long-term sequelae of perinatal asphyxia. Brain Inj 2001;15:1029-39.

8. van Handel M, de Sonneville L, de Vries LS, Jongmans MJ, Swaab H. Specific memory impairment following neonatal encephalopathy in termborn children. Dev Neuropsychol 2012;37:30-50.

9. Marlow N, Rose AS, Rands CE, Draper ES. Neuropsychological and educational problems at school age associated with neonatal encephalopathy. Arch Dis Child Fetal Neonatal Ed 2005;90:F380-7.

10. Azzopardi D, Strohm B, Marlow N, et al.; TOBY Study Group. Effects of hypothermia for perinatal asphyxia on childhood outcomes. N Engl J Med 2014;371:140-9.

11. Deregnier RA, Nelson CA, Thomas KM, Wewerka S, Georgieff MK. Neurophysiologic evaluation of auditory recognition memory in healthy newborn infants and infants of diabetic mothers. J Pediatr 2000;137:777-84.

12. Black LS, deRegnier RA, Long J, Georgieff MK, Nelson CA. Electrographic imaging of recognition memory in 34-38 week gestation intrauterine growth restricted newborns. Exp Neurol 2004;190 Suppl 1:S72-83.

13. deRegnier RA, Wewerka S, Georgieff MK, Mattia F, Nelson CA. Influences of postconceptional age and postnatal experience on the development of auditory recognition memory in the newborn infant. Dev Psychobiol 2002;41:216-25.

14. Mai X, Xu L, Li M, et al. Auditory recognition memory in 2-monthold infants as assessed by event-related potentials. Dev Neuropsychol 2012;37:400-14.

15. Chapman DA, Scott KG, Mason CA. Early risk factors for mental retardation: role of maternal age and maternal education. Am J Ment Retard 2002;107:46-59.

16. Ronfani L, Vecchi Brumatti L, Mariuz M, et al. The complex interaction between home environment, socioeconomic status, maternal IQ and early child neurocognitive development: a multivariate analysis of data collected in a newborn cohort study. PLoS One 2015;10:e0127052.

17. Section on Breastfeeding, American Academy of Pediatrics. Breastfeeding and the use of human milk. Pediatrics 2012;129:e827-e841.

18. Cooper JM, Vargha-Khadem F, Gadian DG, Maguire EA. The effect of hippocampal damage in children on recalling the past and imagining new experiences. Neuropsychologia 2011;49:1843-50.

19. Cooper JM, Gadian DG, Jentschke S, et al. Neonatal hypoxia, hippocampal atrophy, and memory impairment: evidence of a causal sequence. Cereb Cortex 2015;25:1469-76.

20. Tagin MA, Woolcott CG, Vincer MJ, Whyte RK, Stinson DA. Hypothermia for neonatal hypoxic ischemic encephalopathy: an updated systematic review and meta-analysis. Arch Pediatr Adolesc Med 2012;166:558-66.

21. Kwak M, Lim S, Kang E, et al. Effects of neonatal hypoxic-ischemic injury and hypothermic neuroprotection on neural progenitor cells in the mouse hippocampus. Dev Neurosci 2015;37:428-39.

22. Rentería ME. Cerebral asymmetry: a quantitative, multifactorial, and plastic brain phenotype. Twin Res Hum Genet 2012;15:401-13.

23. Perani D, Saccuman MC, Scifo P, et al. Neural language networks at birth. Proc Natl Acad Sci USA 2011;108:16056-61. 


\section{Articles $\mid$ Pfisteretal.}

24. Peña M, Maki A, Kovacić D, et al. Sounds and silence: an optical topography study of language recognition at birth. Proc Natl Acad Sci USA 2003;100:11702-5.

25. Kwon SH, Scheinost D, Lacadie C, et al. Adaptive mechanisms of developing brain: cerebral lateralization in the prematurely-born. Neuroimage 2015;108:144-50.

26. Liang X, Zou Q, He Y, Yang Y. Coupling of functional connectivity and regional cerebral blood flow reveals a physiological basis for network hubs of the human brain. Proc Natl Acad Sci USA 2013;110:1929-34.

27. Vaishnavi SN, Vlassenko AG, Rundle MM, Snyder AZ, Mintun MA, Raichle ME. Regional aerobic glycolysis in the human brain. Proc Natl Acad Sci USA 2010;107:17757-62.

28. Miller SP, Newton N, Ferriero DM, et al. Predictors of 30-month outcome after perinatal depression: role of proton MRS and socioeconomic factors. Pediatr Res 2002;52:71-7.

29. de Haan M. Visual attention and recognition memory in infancy. In: deHaan M. Infant EEG and Event-Related Potentials. London, UK: Psychology Press, 2007:101-109.

30. Glass HC, Glidden D, Jeremy RJ, Barkovich AJ, Ferriero DM, Miller SP. Clinical neonatal seizures are independently associated with outcome in infants at risk for hypoxic-ischemic brain injury. J Pediatr 2009;155: 318-23.

31. Barnett A, Mercuri E, Rutherford M, et al. Neurological and perceptualmotor outcome at 5 - 6 years of age in children with neonatal encephalopathy: relationship with neonatal brain MRI. Neuropediatrics 2002;33:242-8.

32. Belet N, Belet U, Incesu L, et al. Hypoxic-ischemic encephalopathy: correlation of serial MRI and outcome. Pediatr Neurol 2004;31:267-74.

33. Azzopardi D; TOBY study group. Predictive value of the amplitude integrated EEG in infants with hypoxic ischaemic encephalopathy: data from a randomised trial of therapeutic hypothermia. Arch Dis Child Fetal Neonatal Ed 2014;99:F80-2.

34. Padden B, Scheer I, Brotschi B, Wohlrab G, Latal B, Bernet V. Does amplitude-integrated electroencephalogram background pattern correlate with cerebral injury in neonates with hypoxic-ischaemic encephalopathy? J Paediatr Child Health 2015;51:180-5.

35. Sarkar S, Barks JD, Donn SM. Should amplitude-integrated electroencephalography be used to identify infants suitable for hypothermic neuroprotection? J Perinatol 2008;28:117-22.

36. Riggins T, Miller NC, Bauer PJ, Georgieff MK, Nelson CA. Consequences of low neonatal iron status due to maternal diabetes mellitus on explicit memory performance in childhood. Dev Neuropsychol 2009;34:762-79.

37. Nelson CA, Wewerka S, Thomas KM, Tribby-Walbridge S, deRegnier R, Georgieff M. Neurocognitive sequelae of infants of diabetic mothers. Behav Neurosci 2000;114:950-6.

38. van Handel M, de Sonneville L, de Vries LS, Jongmans MJ, Swaab H. Specific memory impairment following neonatal encephalopathy in termborn children. Dev Neuropsychol 2012;37:30-50.

39. Santos J, Pearce SE, Stroustrup A. Impact of hospital-based environmental exposures on neurodevelopmental outcomes of preterm infants. Curr Opin Pediatr 2015;27:254-60.

40. Khedr EM, Farghaly WM, Amry Sel-D, Osman AA. Neural maturation of breastfed and formula-fed infants. Acta Paediatr 2004;93:734-8.

41. Ferguson M, Molfese PJ. Breast-fed infants process speech differently from bottle-fed infants: evidence from neuroelectrophysiology. Dev Neuropsychol 2007;31:337-47.

42. Committee on Fetus and Newborn, Papile LA, Baley JE, et al. Hypothermia and neonatal encephalopathy. Pediatrics. 2014;133:1146-1150.

43. Sarnat HB, Sarnat MS. Neonatal encephalopathy following fetal distress. A clinical and electroencephalographic study. Arch Neurol 1976;33: 696-705.

44. Pfister KM, Gray HL, Miller NC, Demerath EW, Georgieff MK, Ramel SE. Exploratory study of the relationship of fat-free mass to speed of brain processing in preterm infants. Pediatr Res 2013;74:576-83.

45. deRegnier RA. Auditory recognition memory in infancy. In: deHaan M. Infant EEG and Event-Related Potentials. London, UK: Psychology Press, 2007:145-170. 